\title{
Equal-time Consistency Relations in the Large-Scale Structure of the Universe
}

\author{
A. Kehagias ${ }^{a, b}$, H. Perrier $^{b}$ and A. Riotto ${ }^{b}$ \\ a Physics Division, National Technical University of Athens, \\ 15780 Zografou Campus, Athens, Greece \\ ${ }^{b}$ Department of Theoretical Physics and Center for Astroparticle Physics (CAP) \\ 24 quai E. Ansermet, CH-1211 Geneva 4, Switzerland
}

\begin{abstract}
We discuss the consistency relations involving the soft limit of the $(n+1)$-correlator functions of dark matter at equal times and their consequences for the halo model.
\end{abstract}




\section{Introduction}

Symmetry arguments are quite useful in characterizing the cosmological perturbations originated during a de Sitter stage [1]. In particular, the correlators of scalar fields, which are not the inflaton, are constrained by conformal invariance [2-7] as the de Sitter isometry group $\mathrm{SO}(1,4)$ acts like conformal group on $\mathbb{R}^{3}$ when the fluctuations are taken on super-Hubble scales. Conformal consistency relations among the inflationary correlators can also be written if the inflationary perturbations are generated in single-field models of inflation [8-15].

Consistency relations involving the soft limit of the $(n+1)$-correlator functions of matter and galaxy overdensities have been recently derived by exploiting the symmetries of the Newtonian equations of motion of the non-relativistic dark matter and galaxy fluids coupled to gravity $[16,17]$. These consistency relations have been generalized to the relativistic limit [18] (see also [19]), based on the observation that a

long mode, in single-field models of inflation, reduces to a diffeomorphism when it is inside the horizon (as long as it is outside of the sound horizon). Furthermore, these large-scale universe consistency relations have been generalized to redshift space [20,21], their implications for the theories of galaxy bias and modified gravity have been studied in Ref. [20] and their generalization to the case of multi-fluids has been produced in Refs. [22,23].

The soft limit of the $(n+1)$-correlator functions of matter and galaxy overdensities involved in the consistency relations have the property of vanishing in the equal-time limit. This is because they are derived by the basic property that, if an overdensity $\delta(\vec{x}, \tau)$ (say of dark matter) satisfies the fluid equation, then also $\delta^{\prime}(\vec{x}, \tau)=\delta(\vec{x}+\vec{n}(\tau), \tau)$ does (here $\tau$ is the conformal time), where $\vec{n}(\tau)$ is an arbitrary time-dependent vector. Consider the $n$-point correlation function of short modes of the density contrast. The symmetries of the Newtonian fluid equations imply that

$$
\left\langle\delta^{\prime}\left(\vec{x}_{1}\right) \cdots \delta^{\prime}\left(\vec{x}_{n}\right)\right\rangle=\left\langle\delta\left(\vec{x}_{1}\right) \cdots \delta\left(\vec{x}_{n}\right)\right\rangle=\left\langle\delta\left(\vec{x}_{1}^{\prime}\right) \cdots \delta\left(\vec{x}_{n}^{\prime}\right)\right\rangle .
$$

If one now consider $n$-objects contained in a sphere of radius $R$ much smaller than the long wavelength mode of size $\sim 1 / q$ and centered at the origin of the coordinates, one can choose the coordinate transformation in such a way to remove the long and linear wavelength velocity mode $\vec{v}_{L}$. In such a case, Eq. (1.1) is nothing else that the statement that the effect of a physical long wavelength velocity perturbation onto the short modes should be indistinguishable from the long wavelength mode velocity generated by the transformation $[16,17]$

$$
\tau^{\prime}=\tau, \quad \vec{x}^{\prime}=\vec{x}+\vec{n}(\tau)=\vec{x}-\int^{\tau} \mathrm{d} \eta \vec{v}_{L}(\eta, \overrightarrow{0})=\vec{x}+\frac{1}{6} \tau^{2} \vec{\nabla} \Phi_{L}(\eta, \overrightarrow{0}),
$$


where $\Phi_{L}$ is the long wavelength mode of the gravitational potential. Since $(\mathcal{H}=\dot{a} / a$ is the conformal Hubble rate)

$$
\Phi^{\prime}(\vec{x}, \tau)=\Phi\left(\vec{x}^{\prime}, \tau^{\prime}\right)-(\mathcal{H} \dot{\vec{n}}+\ddot{\vec{n}}) \cdot \vec{x}
$$

one is basically removing the homogeneous gravitational force via a change of coordinates. This corresponds to an homogeneous acceleration transformation which allows to go to a free-falling observer, precisely the essence of the Equivalence Principle [18]. In Fourier space, the transformation (1.1) gives for the short non-linear modes

$$
\delta_{\vec{k}}^{\prime}(\tau)=e^{i \vec{k} \cdot \vec{n}(\tau)} \delta_{\vec{k}}(\tau)
$$

and therefore the momentum-conservation $\sum_{i} \vec{k}_{i}=\overrightarrow{0}$ imposes the soft limit of the $(n+1)$-correlator functions of dark matter (as well as galaxy) to vanish at equal time.

The vanishing of the equal-time correlators in the soft limit is therefore rooted in the fact that one can locally eliminate the zero mode and the first spatial gradient of the long and linear wavelength mode of the gravitational potential and that the response of the system on short scales is a uniform displacement.

In this paper, which builds up on previous works [24,25], we discuss what happens if we include two spatial gradients in the game, that is a non-uniform gravitational force. Based on the previous arguments, we expect that the equal-time correlators will not vanish in the soft limit, as short-scale perturbations will now feel the non-homogeneity of the gravitational force. Indeed, we will find a consistency relation for the soft limit of the dark matter correlators which is valid even for non-linear scales and that can be checked against analytical models modeling the clustering of dark matter on short scales.

The paper is organized as follows. In section 2 we discuss the derivation of the equal-time consistency relation, while in section 3 we check it against the halo model. Finally, in section 4 we provide our conclusions.

\section{The equal-time consistency relation for the dark matter bispectrum}

It is well-known that the effect of a long wavelength mode of the gravitational potential of momentum $q$ and including two spatial gradients on the short-scale dynamics is encodable in a local spatial curvature $K=(3 / 5)\left(\delta_{L} / a\right)$ (valid in a matter-dominated universe and after performing an angular average), where $\delta_{L}$ is the linear overdensity [24]. In other words, the physics in a sphere of radius $R \ll 1 / q$ should not 
be distinguishable from the physics with the same curvature as the one induced by the linear mode. To account for the effects of the long wavelength mode there are three effects one should take into account. First of all, the change in scales with respect to the case in which the local system is spatially flat. As the overdensity in the sphere is simply given by

$$
1+\delta_{L}=\frac{a^{3}}{a_{K}^{3}}
$$

where we have indicated by $a$ the scale factor in the region outside the sphere of radius $R$ and by $a_{K}$ the one in the inner region, one simply gets $a / a_{K} \simeq\left(1+\delta_{L} / 3\right)$. This means that comoving momenta are shifted from $\vec{k}$ in the spatially flat outer region to $\vec{k}\left(1-\delta_{L} / 3\right)$ in the inner region.

The second effect is that we have to define the short-mode overdensities with respect to the global average $\bar{\rho}$ and not with respect to the local overdensity $\bar{\rho}\left(1+\delta_{L}\right)$. This brings an extra factor $\left(1+\delta_{L}\right)$ for each density contrast on short scales. Finally, we have to account for the fact that one may trade the time variable of the correlators with the linear growth factor $D(a)$ if no other time dependencies are present in the problem. This is true if $\mathrm{d} \ln D(a) / \mathrm{d} \ln a=\Omega_{\mathrm{m}}^{1 / 2}$ (being $\Omega_{\mathrm{m}}$ the abundance of dar matter with respect to the critical one) which is the condition leading to separability between the density contrast and the peculiar velocity at any order in standard perturbation theory [26]. While not exactly true, the mapping between time and the linear growth factor is good at the $\mathcal{O}(10 \%)$ level for most redshifts [27]. It turns out that in a spatially local closed universe the linear growth factor is enhanced by a factor $\left(1+13 / 21 \delta_{L}\right)$ with respect to the spatially flat case [24]. This is because in a spatially closed universe linear perturbations growth faster than their flat region counterparts if the curvature is positive.

All in all, the dark matter correlation functions on small scales depend on the long wavelength mode as

$$
\begin{aligned}
\xi_{\delta_{L}}(r, a) & \simeq \xi_{0}(r, a) \\
& +2 \delta_{L} \xi_{0}(r, a) \\
& +\frac{1}{3} \delta_{L} r \frac{\partial}{\partial r} \xi_{0}(r, a) \\
& +\frac{13}{21} \delta_{L} \frac{\partial}{\partial \ln D(a)} \xi_{0}(r, a)+\mathcal{O}\left(\delta_{L}^{2}\right)
\end{aligned}
$$

These arguments allow to calculate the soft limit of the three-point correlators of dark matter when the wavenumber $q$ is much smaller than the other two: $k_{1} \simeq k_{2} \simeq 1 / r \gg q$. Indeed, multiplying the result (2.2) by the long wavelength mode $\delta_{L}$, averaging over many realizations and going to momentum space, we obtain 


$$
\left\langle\delta_{\vec{q}}(\tau) \delta_{\vec{k}_{1}}(\tau) \delta_{\vec{k}_{2}}(\tau)\right\rangle_{q \rightarrow 0}^{\prime \text { av }}=P_{L}(q, \tau)\left[1-\frac{1}{3} \frac{\partial}{\partial \ln k_{1}}+\frac{13}{21} \frac{\partial}{\partial \ln D(a)}\right] P\left(k_{1}, \tau\right),
$$

where we have used the fact that

$$
\int \mathrm{d}^{3} x e^{i \vec{k} \cdot \vec{x}} r \frac{\partial}{\partial r} \xi_{0}(r, a)=\left(-3-\frac{\partial}{\partial \ln k}\right) P(k, \tau) .
$$

The label ' av in the bispectrum indicates we have removed the momentum conservation and that the angular average over the angle between the long and the short wavelength modes needs to be taken: in all our considerations we have assumed a spherical average to be able to define a constant spatial curvature to begin with. As expected, the bispectrum does not vanish in the equal-time correlator. The relation (2.3) extends that one found in Ref. [25] which is valid only in the mildly non-linear regime where $P\left(k_{1}, \tau\right) \sim D^{2}(a)$. Of course, the relation (2.3) does not hold in the presence of a primordial non-Gaussianity as the latter introduces an extra correlation among the long and the short wavelength modes.

The generalization of Eq. (2.3) to higher-order correlators is straightforward. Taking into account that there is a factor of $\left(1+\delta_{L} / 3\right)$ for each position vector and a $\left(1+13 / 21 \delta_{L}\right)$ enhancement of the growth factor with respect to the spatially flat case, we get for the equal-time correlator in the long wavelength mode

$$
\begin{aligned}
\left\langle\delta\left(\vec{x}_{1}, \tau\right) \cdots \delta\left(\vec{x}_{n}, \tau\right)\right\rangle_{\delta_{L}} & =\left\langle\delta\left(\vec{x}_{1}, \tau\right) \cdots \delta\left(\vec{x}_{n}, \tau\right)\right\rangle_{0} \\
& +n \delta_{L}\left\langle\delta\left(\vec{x}_{1}, \tau\right) \cdots \delta\left(\vec{x}_{n}, \tau\right)\right\rangle_{0} \\
& +\frac{1}{3} \delta_{L} \sum_{i=1}^{n} \vec{x}_{i} \cdot \nabla_{\vec{x}_{i}}\left\langle\delta\left(\vec{x}_{1}, \tau\right) \cdots \delta\left(\vec{x}_{n}, \tau\right)\right\rangle_{0} \\
& +\frac{13}{21} \delta_{L} \frac{\partial}{\partial \ln D(a)}\left\langle\delta\left(\vec{x}_{1}, \tau\right) \cdots \delta\left(\vec{x}_{n}, \tau\right)\right\rangle_{0}
\end{aligned}
$$

Multiplying the above relation by the long wavelength mode and averaging as before, we get the relation

$$
\left\langle\delta_{\vec{q}}(\tau) \delta\left(\vec{k}_{1}, \tau\right) \cdots \delta\left(\vec{k}_{n}, \tau\right)\right\rangle_{q \rightarrow 0}^{\mathrm{av}}=P_{L}(q, \tau)\left[n-\sum_{i=1}^{n}\left(\frac{1}{3} \frac{\partial}{\partial \ln k_{i}}+1\right)+\frac{13}{21} \frac{\partial}{\partial \ln D(a)}\right]\left\langle\delta\left(\vec{k}_{1}, \tau\right) \cdots \delta\left(\vec{k}_{n}, \tau\right)\right\rangle .
$$

Using the fact that

$$
\sum_{i=1}^{n} \frac{\partial}{\partial \ln k_{i}} \delta\left(\vec{k}_{t}\right)=\vec{k}_{t} \nabla_{\vec{k}_{t}} \delta\left(\vec{k}_{t}\right)
$$


where $\vec{k}_{t}=\vec{k}_{1}+\cdots \vec{k}_{n}$ as well as the general property $f(x) \delta^{\prime}(x)=-\delta(x) f^{\prime}(x)$, we get

$$
\left\langle\delta_{\vec{q}}(\tau) \delta\left(\vec{k}_{1}, \tau\right) \cdots \delta\left(\vec{k}_{n}, \tau\right)\right\rangle_{q \rightarrow 0}^{\prime}=P_{L}(q, \tau)\left[1-\sum_{i=1}^{n} \frac{1}{3} \frac{\partial}{\partial \ln k_{i}}+\frac{13}{21} \frac{\partial}{\partial \ln D(a)}\right]\left\langle\delta\left(\vec{k}_{1}, \tau\right) \cdots \delta\left(\vec{k}_{n}, \tau\right)\right\rangle^{\prime}
$$

Notice that all the considerations made so far are valid for the dark matter overdensities, but not for the galaxies as the trade of the time with the linear growth factor is expected not to hold when including further time dependencies as galaxies form at a range of redshifts and merge. Nevertheless, the result (2.2) should be useful to test analytical models describing the correlation functions of dark matter, as the halo model [28-30]. This is what we proceed to do in the following section.

\section{The equal-time dark matter consistency relation and the halo model}

In its simplest formulation, the halo model assumes that all matter in the universe belongs to dark matter halos, identified by their mass. Therefore, two distinct particles will either belong to the same halo or to two different ones. The power spectrum of density perturbations is the sum of two contributions: the 2-halo term, mainly accounting for the spatial correlations of the distribution of different halos, and the 1-halo term which depends instead on the spatial distribution of matter inside a single halo. Clearly, while the 2-halo term is expected to describe large-scale correlations, the 1-halo term provides predictions in the nonlinear regime. More concretely, the expression for the matter power spectrum is given by

$$
P(k)=P_{2 h}(k)+P_{1 h}(k),
$$

with the 2- and 1-halo contributions given by

$$
\begin{aligned}
& P_{2 h}(k, z)=\frac{1}{\bar{\rho}^{2}}\left[\prod_{i=1}^{2} \int \mathrm{d} m_{i} n\left(m_{i}, z\right) \hat{\rho}\left(k, m_{i}, z\right)\right] P_{h}\left(k, m_{1}, m_{2}\right), \\
& P_{1 h}(k, z)=\frac{1}{\bar{\rho}^{2}} \int \mathrm{d} m n(m, z) \hat{\rho}^{2}(k, m, z),
\end{aligned}
$$

where $n(m)$ is the halo mass function with $n(m) \mathrm{d} m$ the number density of halos of mass between $m$ and $(m+\mathrm{d} m)$, and $\hat{\rho}(k, m, z)$ is the Fourier transform of the spatial density profile $\rho(r, m)$ of a halo of mass $m$ normalized so that $\hat{\rho}(0, m)=m$. The 2-halo term depends as well on the halo power spectrum, $P_{h}\left(k, m_{1}, m_{2}\right)$, describing the correlation between the centers of halos of mass $m_{1}$ and $m_{2}$. As we expect halos to be tracers of the underlying matter distribution, we can assume a linear bias relation between the 
halo and the matter density contrasts, so that $\delta_{h} \approx b_{1} \delta$. Thus, at large scales, the halo power spectrum can be approximated as

$$
P_{h}\left(k, m_{1}, m_{2}\right)=b_{1}\left(m_{1}\right) b_{1}\left(m_{2}\right) P_{L}(k),
$$

where $b_{1}(m)$ represents the linear bias function for halos of mass $m$. Note that for Gaussian initial conditions, $b_{1}$ only depends on the mass $m$, as implicitly assumed in Eq. (3.4). The 2-halo term can be rewritten as

$$
P_{2 h}(k, z)=\frac{1}{\bar{\rho}^{2}}\left[\prod_{i=1}^{2} \int \mathrm{d} m_{i} n\left(m_{i}, z\right) \hat{\rho}\left(k, m_{i}, z\right) b_{1}(m, z)\right] P_{L}(k) .
$$

This description can be easily extended to the matter bispectrum. In the case of a three-point function, we should account for the possibility that the three points belong to just one, two or three dark matter halos. This means that there are now three distinct contributions to the halo model expression for the matter bispectrum, that is

$$
\left\langle\delta_{\vec{k}_{1}}(\tau) \delta_{\vec{k}_{2}}(\tau) \delta_{\vec{k}_{3}}(\tau)\right\rangle \equiv B\left(k_{1}, k_{2}, k_{3}\right)=B_{3 h}\left(k_{1}, k_{2}, k_{3}\right)+B_{2 h}\left(k_{1}, k_{2}, k_{3}\right)+B_{1 h}\left(k_{1}, k_{2}, k_{3}\right),
$$

where

$$
\begin{aligned}
B_{3 h}\left(k_{1}, k_{2}, k_{3}, z\right)= & \frac{1}{\bar{\rho}^{3}}\left[\prod_{i=1}^{3} \int \mathrm{d} m_{i} n\left(m_{i}, z\right) \hat{\rho}\left(m_{i}, z, k_{i}\right)\right] B_{h}\left(k_{1}, m_{1} ; k_{2}, m_{2} ; k_{3}, m_{3} ; z\right), \\
B_{2 h}\left(k_{1}, k_{2}, k_{3}, z\right)= & \frac{1}{\bar{\rho}^{3}} \int \mathrm{d} m n(m, z) \hat{\rho}\left(m, z, k_{1}\right) \int \mathrm{d} m^{\prime} n\left(m^{\prime}, z\right) \hat{\rho}\left(m^{\prime}, z, k_{2}\right) \hat{\rho}\left(m^{\prime}, z, k_{3}\right) \\
& \times P_{h}\left(k_{1}, m, m^{\prime}, z\right)+\text { cyc. }, \\
B_{1 h}\left(k_{1}, k_{2}, k_{3}, z\right)= & \frac{1}{\bar{\rho}^{3}} \int \mathrm{d} m n(m, z) \hat{\rho}\left(k_{1}, m, z\right) \hat{\rho}\left(k_{2}, m, z\right) \hat{\rho}\left(k_{3}, m, z\right) .
\end{aligned}
$$

In this case, while the 2-halo term depends on the halo power spectrum as in the previous case, the 3halo term involves the halo bispectrum, $B_{h}\left(k_{1}, m_{1} ; k_{2}, m_{2} ; k_{3}, m_{3} ; z\right)$. Assuming again a local bias relation between halos and matter, $\delta_{h}(m)=f(\delta)$, expanded perturbatively as $\delta_{h}(m)=b_{1}(m) \delta+\left[b_{2}(m) / 2\right] \delta^{2}+$ $\mathcal{O}\left(\delta^{3}\right)$, it is possible to derive the tree-level expression for the halo bispectrum, valid only in the large-scale limit, in terms of the matter power spectrum $P(k)$ and the bispectrum $B\left(k_{1}, k_{2}, k_{3}\right)$. This reads

$$
\begin{aligned}
B_{h}\left(k_{1}, m_{1} ; k_{2}, m_{2} ; k_{3}, m_{3} ; z\right)= & b_{1}\left(m_{1}\right) b_{1}\left(m_{2}\right) b_{1}\left(m_{3}\right) B\left(k_{1}, k_{2}, k_{3}\right) \\
& +\left[b_{1}\left(m_{1}\right) b_{1}\left(m_{2}\right) b_{2}\left(m_{3}\right) P\left(k_{1}\right) P\left(k_{2}\right)+\text { cyc. }\right],
\end{aligned}
$$

where $b_{2}(m)$ is the quadratic bias function. For Gaussian initial conditions $b_{1}$ and $b_{2}$ are scale independent. Moreover, since this equation is valid on large scales, we can replace the matter power spectrum $P$ by its linear prediction $P_{L}$ and the matter bispectrum $B$ by its gravitational contribution $B_{\mathrm{G}}$ 


$$
B_{\mathrm{G}}\left(k_{1}, k_{2}, k_{3}\right)=2 F_{2}\left(\vec{k}_{1}, \vec{k}_{2}\right) P_{L}\left(k_{1}\right) P_{L}\left(k_{2}\right)+2 \text { perm., }
$$

$F_{2}$ being the standardl kernel representing the second-order solution in perturbation theory and given by

$$
F_{2}\left(\vec{k}_{i}, \vec{k}_{j}\right)=\frac{5}{7}+\frac{1}{2}\left(\frac{k_{i}}{k_{j}}+\frac{k_{j}}{k_{i}}\right)\left(\hat{k}_{i} \cdot \hat{k}_{j}\right)+\frac{2}{7}\left(\hat{k}_{i} \cdot \hat{k}_{j}\right)^{2}
$$

The spatial distribution of matter in a halo of mass $m$ is specified by the halo density profile $\rho(r, m)$, interpreted as an average over all halos of the same mass. We consider the Navarro, Frenk and White form for the halo density profile [31] $\rho(r)=\rho_{s} /\left[\left(r / r_{s}\right)\left(1+r / r_{s}\right)^{2}\right]$, which assumes a universal profile as a function of $r$. The parameters $r_{s}$ and $\rho_{s}$ can be expressed in terms of the virial mass of the halo $m$ and the concentration parameter $c$. In particular, the virial mass is given by $m \equiv(4 \pi / 3) R_{v}^{3} \Delta_{v} \bar{\rho}$, with $R_{v}$ the virial radius, defined as the radius of a sphere within which the mean density of the halo is $\Delta_{v}$ times that of the universe. We take $\Delta_{v}=200$. The concentration parameter $c$ is defined as $c=R_{v} / r_{s}$ and is typically a function of $m$. The halo mass function $n(m)$ [32] characterizes the number density of halos per unit mass. The fraction of the total mass of the universe contained in all the halos with mass in the range $m$ and $(m, m+\mathrm{d} m)$ can be written as

$$
\frac{1}{\bar{\rho}} n(m) m \mathrm{~d} m=f(\nu) \mathrm{d} \nu
$$

The function $f(\nu)$ has an approximately universal form and depends on the variable

$$
\nu \equiv \frac{\delta_{\mathrm{c}}}{\sigma(m, z)},
$$

with $\delta_{\mathrm{c}}$ representing the critical density for spherical collapses (we assume the Einstein-de Sitter value $\delta_{\mathrm{c}}=1.68$ at zero redshift) while $\sigma(m, z)=D(z) \sigma(m, z=0)$ is the square root of the variance of matter fluctuations in spheres of radius $R=(3 m / 4 \pi \bar{\rho})^{1 / 3}$ (associated to the Fourier transform $W_{R}(k)$ of the top-hat function in real space), $\sigma^{2}(m) \equiv 4 \pi \int \mathrm{d} k k^{2} P_{L}(k) W_{R}(k)$. We will adopt the Sheth and Tormen form of the halo mass function [33] expression

$$
\nu f(\nu)=A \sqrt{\frac{a \nu^{2}}{2 \pi}}\left[1+\frac{1}{\left(a \nu^{2}\right)^{p}}\right] e^{-a \nu^{2} / 2},
$$

where $a=0.707$ and $p=0.3$ while $A=0.322$ ensures a proper normalization. Finally, the bias functions can be derived from the unconditional halo mass function and, in the case of the Sheth-Tormen form, one obtains for the first two the expressions [33]

$$
\begin{aligned}
& b_{1}(\nu)=1+\frac{a \nu^{2}-1}{\delta_{\mathrm{c}}}+\frac{2 p}{\delta_{\mathrm{c}}\left(1+\left(a \nu^{2}\right)^{p}\right)} \\
& b_{2}(\nu)=\frac{8}{21}\left[b_{1}(\nu)-1\right]+\frac{a \nu^{2}}{\delta_{\mathrm{c}}} \frac{a \nu^{2}-3}{\delta_{\mathrm{c}}}+\left(\frac{1+2 p}{\delta_{\mathrm{c}}}+2 \frac{a \nu^{2}-1}{\delta_{\mathrm{c}}}\right) \frac{2 p / \delta_{\mathrm{c}}}{1+\left(a \nu^{2}\right)^{p}} .
\end{aligned}
$$


The requirement for the total matter density to be given by

$$
\rho(\vec{x}) \equiv \bar{\rho}[1+\delta(\vec{x})]=\int \mathrm{d} m m n(m)\left[1+\sum_{i} \frac{b_{i}(m)}{i !} \delta^{i}(\vec{x})\right],
$$

imposes the condition

$$
\int \mathrm{d} m m n(m)=\bar{\rho}
$$

along with the constraints on the bias functions,

$$
\frac{1}{\bar{\rho}} \int \mathrm{d} m m n(m) b_{i}(m)=\int \mathrm{d} \nu f(\nu) b_{i}(\nu)=\delta_{i 1} .
$$

Such relations assure that, on large scales $(k \rightarrow 0, \hat{\rho} \rightarrow m)$, the 2-halo term of the power spectrum reduces to the linear power spectrum and the 3-halo term of the bispectrum reduces to the large-scale matter bispectrum.

We are now ready to study the squeezed limit of the bispectrum. It allows for a significant simplification of the halo model expressions. As physical intuition dictates, in this limit the largest contribution to the bispectrum comes from the position-space configuration where two points are close and belong to the same halo while the third one is at larger distance from the first two, and hence is likely to belong to another halo. In this case we expect the halo model prediction to be dominated by the 2-halo contribution, with the 1-halo and 3-halo terms being subdominant. This expectation is confirmed numerically [34]: taking the smallest wavenumber to be $q=0.014 h \mathrm{Mpc}^{-1}, B_{2 h}$ becomes dominant over the other two terms for $k_{1} \sim k_{2} \sim 0.3 h \mathrm{Mpc}^{-1}$ and at $k_{1} \sim k_{2} \sim 1 h \mathrm{Mpc}^{-1}$ the sum of $B_{1 h}$ and $B_{3 h}$ contribute less than $10 \%$.

If $q$ is still in the linear regime, then we can safely set that the Fourier transform of the halo profile to be

$$
\hat{\rho}(m, z, q) \simeq m
$$

in all bispectrum terms involving $q$ in Eqs. (3.7), (3.8) and (3.9). By making this substitution, the expressions for the 1-, 2- and 3-halo terms greatly simplify. Moreover, by using the conditions in Eqs. (3.19) and (3.20), together with Eq. (3.21), the halo model bispectrum contributions become, at leading order in $q[34]$

$$
\begin{aligned}
& B_{1 h}\left(q, k_{1}, k_{2}\right)=\frac{1}{\bar{\rho}} \epsilon_{2}^{[m]}\left(k_{1}\right) \\
& B_{2 h}\left(q, k_{1}, k_{2}\right)=\epsilon_{2}^{\left[b_{1}\right]}\left(k_{1}\right) P_{L}(q) \\
& B_{3 h}\left(q, k_{1}, k_{2}\right)=2\left[\frac{13}{14}+\left(\frac{4}{7}-\frac{1}{2} \frac{\partial \ln P_{L}}{\partial \ln k_{1}}\right)\left(\hat{q} \cdot \hat{k}_{1}\right)^{2}+\frac{\epsilon_{1}^{\left[b_{2}\right]}\left(k_{1}\right)}{\epsilon_{1}^{\left[b_{1}\right]}\left(k_{1}\right)}\right] P_{L}(q) P_{2 h}\left(k_{1}\right) .
\end{aligned}
$$


The functions $\epsilon_{i}^{[F]}$ in these expressions are defined as

$$
\epsilon_{i}^{[F]}(k) \equiv \frac{1}{\bar{\rho}^{i}} \int \mathrm{d} m n(m, z) \hat{\rho}^{i}(m, z, k) F(m, z),
$$

where $F(m, z)$ represents a generic function of mass and redshift. Thus, these functions are like an "average" of the function $F$, weighted by the mass function and the $i$-th power of the Fourier transform of the density profile. The first two terms inside the bracket of Eq. (3.24) have been derived by taking the squeezed limit $q \ll k_{1} \simeq k_{2}$ of $B_{\mathrm{G}}\left(k_{1}, k_{2}, k_{3}\right)$ in Eq. (3.11)

$$
\begin{aligned}
B_{\mathrm{G}}\left(q, k_{1}, k_{2}\right) & \simeq 2\left[F_{2}\left(\vec{q}, \vec{k}_{1}\right) P_{L}(q) P_{L}\left(k_{1}\right)+F_{2}\left(\vec{q}, \vec{k}_{2}\right) P_{L}(q) P_{L}\left(k_{2}\right)\right] \\
& =2\left[\frac{13}{14}+\left(\frac{4}{7}-\frac{1}{2} \frac{\partial \ln P_{L}}{\partial \ln k_{1}}\right)\left(\hat{q}_{1} \cdot \hat{k}_{1}\right)^{2}+\mathcal{O}\left(q / k_{1}\right)\right] P_{L}(q) P_{L}\left(k_{1}\right) .
\end{aligned}
$$

Let us first consider the angular averaged of the squeezed limit of the bispectrum in the limited range of momenta where it is dominated by the 3-halo piece, that is in the limit in which all momenta are in the linear regime, and one can safely take $P\left(k_{1}\right) \simeq P_{2 h}\left(k_{1}\right) \simeq P_{L}\left(k_{1}\right)$. In such a case

$$
\begin{aligned}
\left\langle\delta_{\vec{q}}(\tau) \delta_{\vec{k}_{1}}(\tau) \delta_{\vec{k}_{2}}(\tau)\right\rangle_{q \rightarrow 0}^{\prime \text { av }} & \simeq 2\left[\frac{13}{14}+\frac{1}{3} \cdot \frac{4}{7}-\frac{1}{6} \frac{\partial \ln P_{L}}{\partial \ln k_{1}}+\frac{\epsilon_{1}^{\left[b_{2}\right]}\left(k_{1}\right)}{\epsilon_{1}^{\left[b_{1}\right]}\left(k_{1}\right)}\right] P_{L}(q) P_{2 h}\left(k_{1}\right) \\
& \simeq\left[\frac{47}{21}-\frac{1}{3} \frac{\partial}{\partial \ln k_{1}}+2 \frac{\epsilon_{1}^{\left[b_{2}\right]}\left(k_{1}\right)}{\epsilon_{1}^{\left[b_{1}\right]}\left(k_{1}\right)}\right] P_{L}(q) P\left(k_{1}\right) .
\end{aligned}
$$

This has to be compared with the prediction of the consistency relation (2.3) which gives, taking into account that in the present case $P\left(k_{1}\right)=P_{L}\left(k_{1}\right) \propto D^{2}(a)$,

$$
\left\langle\delta_{\vec{q}}(\tau) \delta_{\vec{k}_{1}}(\tau) \delta_{\vec{k}_{2}}(\tau)\right\rangle_{q \rightarrow 0}^{\prime \text { av }} \simeq\left[\frac{47}{21}-\frac{1}{3} \frac{\partial}{\partial \ln k_{1}}\right] P_{L}(q) P\left(k_{1}\right)
$$

Since in the limit we are taking

$$
\begin{aligned}
\epsilon_{1}^{\left[b_{2}\right]}\left(k_{1}\right) & =\frac{1}{\bar{\rho}} \int \mathrm{d} m m n(m) b_{2}\left(m, k_{1}\right)=0, \\
\epsilon_{1}^{\left[b_{1}\right]}\left(k_{1}\right) & =\frac{1}{\bar{\rho}} \int \mathrm{d} m m n(m) b_{1}\left(m, k_{1}\right)=1,
\end{aligned}
$$

we conclude that the halo model prediction (3.27) reproduces the consistency relation.

Let us now consider the most realistic case in which the momenta $k_{1}$ and $k_{2}$ are in the non-linear regime. In such a case, the bispectrum is dominated by the 2-halo piece and one gets 


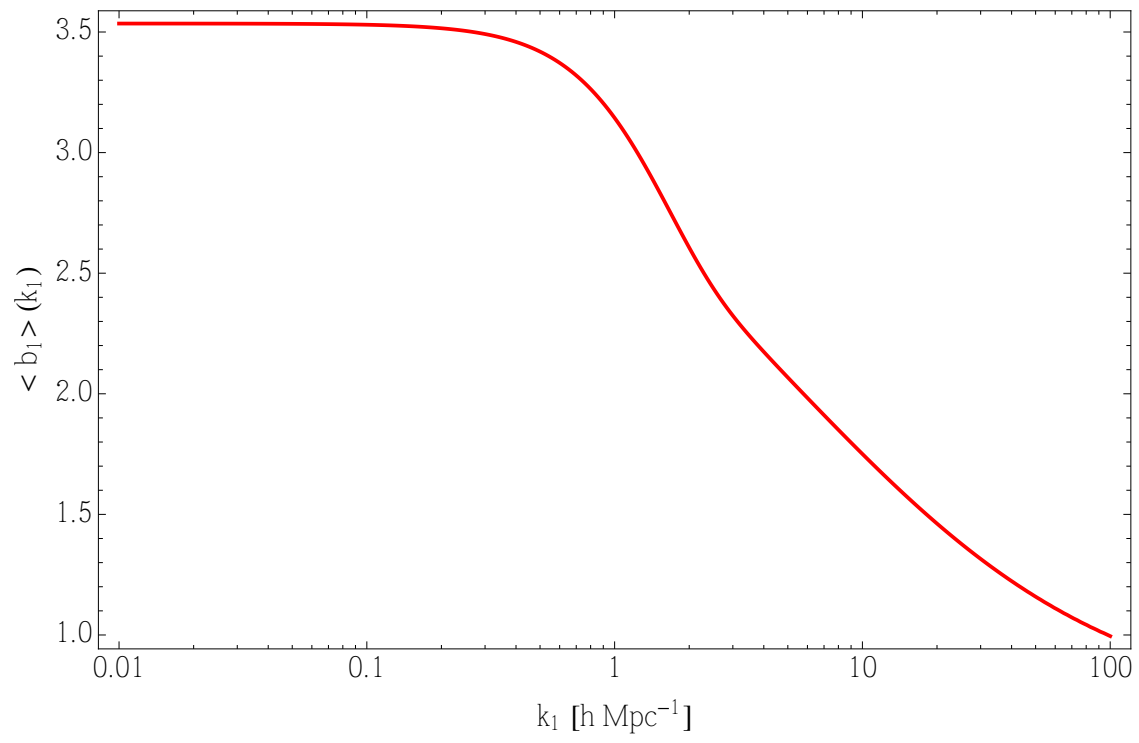

Figure 1: The function $\left\langle b_{1}\right\rangle\left(k_{1}\right)$ as a function of $k_{1}$.

$$
\begin{aligned}
\left\langle\delta_{\vec{q}}(\tau) \delta_{\vec{k}_{1}}(\tau) \delta_{\vec{k}_{2}}(\tau)\right\rangle_{q \rightarrow 0}^{\prime \text { av }} & \simeq P_{L}(q) P_{1 h}\left(k_{1}\right) \frac{\int \mathrm{d} m n(m, z) \hat{\rho}^{2}\left(m, z, k_{1}\right) b_{1}\left(k_{1}\right)}{\int \mathrm{d} m n(m, z) \hat{\rho}^{2}\left(m, z, k_{1}\right)} \\
& =\left\langle b_{1}\right\rangle\left(k_{1}\right) P_{L}(q) P_{1 h}\left(k_{1}\right)
\end{aligned}
$$

where the ratio of the two integrals provides an average of the first bias parameter. We see from Fig. 1 that $\left\langle b_{1}\right\rangle\left(k_{1}\right)$ is constant up to $k_{1}=\mathcal{O}(1) h \mathrm{Mpc}^{-1}$ and its value is approximately 3.5. At much larger values of $k_{1}$ the average $\left\langle b_{1}\right\rangle\left(k_{1}\right)$ acquires a strong momentum dependence. To check if the analytical prediction of the halo models satisfies the consistency relation we need to compute the 1-halo power spectrum. At large values of $k_{1}$ the power spectrum is dominated by contributions near the mass scale for which $k_{1} r_{s} \simeq 1$ [29]. The mass function behaves as $\mathrm{d} n / \mathrm{d} m \sim m^{-2} \nu^{1-2 p} \exp \left(-a \nu^{2} / 2\right)$. Since the scale radius $r_{s}$ depends on the mass as $r_{s}=R_{v} / c \sim m^{1 / 3} / m^{-(3+n) / 6}=m^{(5+n) / 6}$, where $n$ is the spectral index of the linear power spectrum, we find that the power spectrum at high momenta goes as [29]

$$
P_{1 h}\left(k_{1}\right) \sim \int \mathrm{d} m \nu^{1-2 p} \hat{\rho}^{2}\left(k_{1} r_{s}\right)
$$

We change variables to $x=k_{1} r_{s} \sim k_{1}\left[m / m_{*}(z)\right]^{(5+n) / 6}$, where we have defined $m_{*}(z)$ the mass scale for which $\sigma\left[\left(m_{*}(z)\right]=1\right.$. Accounting for the fact that $\sigma(m) \sim\left(m / m_{*}\right)^{-(3+n) / 6}$, we get 


$$
P_{1 h}\left(k_{1}\right) \sim[D(a)]^{\frac{6}{n+3}+1-2 p} k^{\gamma-3}, \quad \gamma=\frac{9+3 n}{5+n}-(1-2 p)\left(\frac{3+n}{5+n}\right) .
$$

The consistency relation (2.3) therefore would give

$$
\left\langle\delta_{\vec{q}}(\tau) \delta_{\vec{k}_{1}}(\tau) \delta_{\vec{k}_{2}}(\tau)\right\rangle_{q \rightarrow 0}^{\prime \text { av }} \simeq\left[1-\frac{1}{3}(\gamma-3)+\frac{13}{21}\left(\frac{6}{n+3}+1-2 p\right)\right] P_{L}(q) P_{1 h}\left(k_{1}\right),
$$

which seems reproduce the result (3.30) quite remarkably. For instance, for $n \simeq-1$, one gets a coefficient of order of 3.6 in Eq. (3.33) in front of $P_{L}(q) P_{1 h}\left(k_{1}\right)$. On general grounds, as the spectral index of the short-mode power spectrum is negative and the dependence on the growth factor is such that the non-linear power spectrum is suppressed at high redshifts, implying a positive power of $D(a)$, this leads to the conclusion that the overall coefficient in front of $P_{L}(q) P_{1 h}\left(k_{1}\right)$ in the bispectrum is predicted to be larger than unity by the consistency relation. This is in good agreement with the prediction of the halo model: the halo model reproduces the remarkable property predicted by the consistency relation that the bispectrum in the squeezed limit factorizes in terms of the product of the linear power spectrum times the non-linear one. This conclusion seems to be valid up to scales $\mathcal{O}(1) h \mathrm{Mpc}^{-1}$.

\section{Conclusion}

In this paper we have derived a consistency relation which applies for dark matter overdensities and involves the soft limit of the $(n+1)$-point correlation functions. While this result can be hardly extended to the more interesting case of galaxy overdensities, we have used it to investigate the ability of the halo model to satisfy the consistent relations. We have concluded that the halo model satisfies rather well the features predicted by the consistency relation up to scales $\mathcal{O}(1) h \mathrm{Mpc}^{-1}$.

\section{Acknowledgments}

When completing this work, Ref. [35] appeared. Our results agree with those contained in this reference. We thank D. Figueroa for useful numerical assistance. A.R. is supported by the Swiss National Science Foundation (SNSF), project 'The non-Gaussian universe" (project number: 200021140236). The research of A.K. was implemented under the "Aristeia" Action of the "Operational Programme Education and Lifelong Learning" and is co-funded by the European Social Fund (ESF) and National Resources. It is partially supported by European Union's Seventh Framework Programme (FP7/2007-2013) under REA grant agreement n. 329083. 


\section{References}

[1] D. H. Lyth and A. Riotto, Phys. Rept. 314, 1 (1999) [hep-ph/9807278].

[2] I. Antoniadis, P. O. Mazur and E. Mottola, JCAP 1209, 024 (2012) [arXiv:1103.4164 [gr-qc]].

[3] J. M. Maldacena and G. L. Pimentel, JHEP 1109, 045 (2011) [arXiv:1104.2846 [hep-th]].

[4] P. Creminelli, Phys. Rev. D 85, 041302 (2012) [arXiv:1108.0874 [hep-th]].

[5] A. Kehagias and A. Riotto, Nucl. Phys. B 864, 492 (2012) [arXiv:1205.1523 [hep-th]].

[6] A. Kehagias and A. Riotto, Nucl. Phys. B 868, 577 (2013) [arXiv:1210.1918 [hep-th]].

[7] M. Biagetti, A. Kehagias, E. Morgante, H. Perrier and A. Riotto, JCAP 07, 030 (2013) [arXiv:1304.7785 [astro-ph.CO]].

[8] P. Creminelli, J. Noreña and M. Simonović, JCAP 1207, 052 (2012) [arXiv:1203.4595 [hep-th]].

[9] K. Hinterbichler, L. Hui and J. Khoury, JCAP 1208, 017 (2012) [arXiv:1203.6351 [hep-th]].

[10] V. Assassi, D. Baumann and D. Green, JCAP 1211, 047 (2012) [arXiv:1204.4207 [hep-th]].

[11] V. Assassi, D. Baumann and D. Green, JHEP 1302, 151 (2013) [arXiv:1210.7792 [hep-th]].

[12] A. Bzowski, P. McFadden and K. Skenderis, JHEP 1304, 047 (2013) [arXiv:1211.4550 [hep-th]].

[13] I. Mata, S. Raju and S. Trivedi, JHEP 1307, 015 (2013) [arXiv:1211.5482 [hep-th]].

[14] K. Hinterbichler, L. Hui and J. Khoury, arXiv:1304.5527 [hep-th].

[15] L. Berezhiani and J. Khoury, arXiv:1309.4461 [hep-th].

[16] A. Kehagias and A. Riotto, Nucl. Phys. B 873, 514 (2013) [arXiv:1302.0130 [astro-ph.CO]].

[17] M. Peloso and M. Pietroni, JCAP 1305, 031 (2013) [arXiv:1302.0223 [astro-ph.CO]].

[18] P. Creminelli, J. Noreña, M. Simonović and F. Vernizzi, arXiv:1309.3557 [astro-ph.CO].

[19] A. Kehagias and A. Riotto, arXiv:1309.3671 [hep-th].

[20] A. Kehagias, J. Noreña, H. Perrier and A. Riotto, arXiv:1311.0786 [astro-ph.CO].

[21] P. Creminelli, J. Gleyzes, M. Simonović and F. Vernizzi, arXiv:1311.0290 [astro-ph.CO]. 
[22] M. Peloso and M. Pietroni, arXiv:1310.7915 [astro-ph.CO].

[23] P. Valageas, arXiv:1311.1236 [astro-ph.CO].

[24] See, for instance, T. Baldauf, U. Seljak, L. Senatore and M. Zaldarriaga, JCAP 1110, 031 (2011) [arXiv:1106.5507 [astro-ph.CO]].

[25] B. D. Sherwin and M. Zaldarriaga, Phys. Rev. D 85, 103523 (2012) [arXiv:1202.3998 [astro-ph.CO]].

[26] F. Bernardeau, S. Colombi, E. Gaztanaga and R. Scoccimarro, Phys. Rept. 367, 1 (2002) [astro$\mathrm{ph} / 0112551]$.

[27] M. Pietroni, JCAP 0810, 036 (2008) [arXiv:0806.0971 [astro-ph]].

[28] U. Seljak, Mon. Not. Roy. Astron. Soc. 318, 203 (2000) [astro-ph/0001493].

[29] C. -P. Ma and J. N. Fry, Astrophys. J. 543, 503 (2000) [astro-ph/0003343].

[30] A. Cooray and R. K. Sheth, Phys. Rept. 372, 1 (2002) [astro-ph/0206508].

[31] J. F. Navarro, C. S. Frenk and S. D. M. White, Astrophys. J. 490, 493 (1997) [astro-ph/9611107].

[32] For a review, see A. R. Zentner, Int. J. Mod. Phys. D 16, 763 (2007) [astro-ph/0611454].

[33] R. K. Sheth and G. Tormen, Mon. Not. Roy. Astron. Soc. 308, 119 (1999) [astro-ph/9901122].

[34] D. G. Figueroa, E. Sefusatti, A. Riotto and F. Vernizzi, JCAP 1208, 036 (2012) [arXiv:1205.2015 [astro-ph.CO]].

[35] P. Valageas, arXiv:1311.4286 [astro-ph.CO]. 